\title{
Anlotinib Combined with Cranial Radiotherapy for Non-Small Cell Lung Cancer Patients with Brain Metastasis: A Retrospectively, Control Study
}

\author{
Zelai $\mathrm{He}^{\mathrm{l}, *}$ \\ Jia Liu',* \\ Yuwei Mal,* \\ Hao Jiang' \\ Zhen Cui ${ }^{1}$ \\ Guowen Wang' \\ Yufeng Wu $\mathbb{1}^{2}$ \\ Jiuzhou Liu $^{3}$ \\ Xixi $\mathrm{Cai}^{4}$ \\ Jing Qian' \\ Jingwen Huang' \\ Huijun Zhang ${ }^{5}$ \\ Hongwei $\mathrm{Li}^{1}$
}

'The First Affiliated Hospital of Bengbu Medical College \& Tumor Hospital Affiliated to Bengbu Medical College, Bengbu, 233004, People's Republic of China; ${ }^{2}$ Department of Internal Medicine, Affiliated Cancer Hospital of Zhengzhou University, Henan Cancer Hospital, Zhengzhou, Henan, 450008, People's Republic of China; ${ }^{3}$ The First Affiliated Hospital of Henan University of Science and Technology, Luoyang, 47I003,

People's Republic of China; ${ }^{4}$ Department of Radiation Oncology, The Second Affiliated Hospital of Bengbu Medical College, Bengbu, 233004, People's Republic of China; ${ }^{5}$ Department of Cardiothoracic Surgery, Huashan Hospital of Fudan University, Shanghai, 200040, People's Republic of China

*These authors contributed equally to this work

Correspondence: Huijun Zhang; Hongwei Li

Email zhanghuijunhp@।63.com;

|249223318@qq.com
Introduction: Cranial radiotherapy (CRT) is the main treatment for non-small cell lung cancer (NSCLC) with brain metastasis (BM) and non-EGFR/ALK/ROS1-TKIs indication, and anlotinib can improve overall prognosis. However, the clinical effects of CRT combined with anlotinib for the treatment of NSCLC with BM remain unclear.

Methods: We retrospectively analyzed the clinical effects of anlotinib + CRT versus CRT alone in NSCLC patients with BM and non-EGFR/ALK/ROS1-TKIs indication from September 2016 to June 2020. The progression-free survival (PFS) and overall survival (OS) of anlotinib + CRT versus CRT alone were analyzed. After evaluation of the clinical characteristics to generate a baseline, the independent prognostic factors for intracranial PFS (iPFS) and OS were subjected to univariate and multivariate analysis. Finally, subgroup analysis for iPFS and OS was performed to assess treatment effects using randomized stratification factors and stratified Cox proportional hazards models.

Results: This study included data for 73 patients with BM at baseline. Of the 73 patients, 45 patients received CRT alone, and 28 patients received CRT + anlotinib. There was no significant difference in clinical features between the two groups $(P>0.05)$. Compared with the CRT group, the combined group had longer iPFS (median iPFS [miPFS]: 3.0 months vs 11.0 months, $P=0.048$ ). However, there were no significant differences in OS, extracranial PFS, and systemic PFS. For clinical features, univariate and multivariate analysis showed that the plus anlotinib treatment was an independent advantage predictor of iPFS (hazard ratio [HR] $0.51 ; 95 \%$ confidence interval [CI] $0.27-0.95 ; P=0.04$ ), and age $\geq 57$ years (HR 1.04, 95\% CI 1.01-1.08, $P=0.014$ ) and KPS score $\leq 80$ (HR $1.04,95 \%$ CI $1.01-$ $1.08, P=0.014)$ were independent disadvantage predictors of $\mathrm{OS}(P<0.05)$. In addition, although this difference was not statistically significant $(p>0.05)$, the patients with the anlotinib + local CRT (LCRT) treatment had the longest iPFS (miPFS: 27.0 months) and OS (median OS [mOS]: 36 months). The miPFS and mOS values for the LCRT group were 11 months and 18 months, respectively, with shorter values for whole-brain RT (WBRT) + anlotinib group, WBRT + LCRT + anlotinib group, WBRT, and WBRT + LCRT.

Conclusion: Anlotinib can improve the intracranial lesion control and survival prognosis of NSCLC patients with CRT.

Keywords: radiotherapy, lung cancer, brain metastases, progression-free survival, overall survival

\section{Background}

Lung cancer is the most common of all malignant tumors worldwide. ${ }^{1-3}$ The proportion of non-small cell lung cancer (NSCLC) in all lung cancer is about $80 \%$, and $30-43 \%$ of patients will have brain metastasis (BM) in the process of 
the disease. ${ }^{4}$ The prognosis of lung cancer patients with $\mathrm{BM}$ is poor, and the median overall survival (mOS) of patients that do not receive treatment is only 1-3 months. ${ }^{5}$

In recent years, significantly improved prognosis has been achieved for lung cancer patients by treatment using small molecular targeted tyrosine kinase inhibitors (TKIs) to target anaplastic lymphoma kinase (ALK), epidermal growth factor receptor (EGFR), and C-ros oncogene 1receptor tyrosine kinase (ROS1). ${ }^{6}$ Patients with $\mathrm{BM}$ from NSCLC receiving these treatments also showed significantly improved survival. ${ }^{7}$ However, for advanced NSCLC patients with non-gene mutation or resistance to EGFR/ALK/ROS1-TKIs, alternative TKIs are not available, making it urgent to develop specific targeted drugs.

Anlotinib hydrochloride was independently developed in China as an orally administered, multi-target TKI. ${ }^{8}$ Anlotinib can inhibit tumor cell proliferation and tumor angiogenesis by inhibiting tumor-related kinases, such as VEGFR, FGFR, PDGFR $\alpha / \beta$, c-kit, and RET. ${ }^{8-10}$ In ALTER1202, ALTER0302 and ALTER0303 trials, the overall survival (OS) and progression-free survival (PFS) of the anlotinib group were significantly better than that of the placebo $(p<0.05))^{5,10-12}$ The ALTER0303 clinical trial evaluated the efficacy of anlotinib for the treatment of BM. For these patients with BM at baseline, the mPFS for anlotinib treatment was 4.17 months, considerably higher than the 1.3 months for placebo treatment, and the mOS for anlotinib treatment was 8.57 months compared to 4.55 months for placebo treatment. The patients in the anlotinib group exhibited a longer time to brain progression (TTBP) than the placebo group, indicating that anlotinib delays the progression of intracranial lesions from advanced NSCLC patients with non-gene mutation or resistance to EGFR/ALK/ROS1-TKIs. ${ }^{12}$ Therefore, in May 2018, the China Food and Drug Administration officially approved anlotinib for third-line or higher treatment of advanced NSCLC patients with non-EGFR/ALK/ ROS1-TKIs indication.

Clinical studies have confirmed that anlotinib can effectively treat some patients with advanced lung cancer, including patients with $\mathrm{BM}^{12}$ However, for NSCLC patients with no specific gene mutation or EGFR/ALK/ ROS1-TKIs resistance, cranial radiotherapy (CRT) is still considered the standard treatment regime, as this treatment can quickly relieve central nervous system symptoms and improve the survival time of patients. ${ }^{13}$ CRT can increase the permeability of the blood-brain barrier (BBB), ${ }^{14}$ which may increase anlotinib content in brain tissue, so the curative effect of CRT combined with anlotinib may be better than that of CRT alone for NSCLC patients with no specific gene mutation or EGFR/ALK/ROS1-TKIs resistance. In this study, we retrospectively analyzed the treatment effects of CRT combined with anlotinib compared with CRT alone for patients with lung cancer BM and multi-line chemotherapy failure or patients with EGFR/ ALK/ROS1-TKIs resistance or patients with non-EGFR/ ALK/ROS1 mutations or intolerable chemotherapy.

\section{Methods}

\section{Patients}

We reviewed the clinical records of patients diagnosed with NSCLC and BM between September 2016 and June 2020 at The First Affiliated Hospital of Bengbu Medical College (China). The clinical records of these patients included their clinical information, imaging data, tumorrelated features, treatment process and clinical outcomes. Clinical information included gender, age, smoking and drinking history, previous disease history, and Karnofsky Performance Status (KPS) score. Tumor-related features included pathological type, EGFR/ALK/ROS1 mutation status, extracranial metastasis, number of $\mathrm{BM}$, and treatment process (including CRT and drug therapy). ${ }^{12}$ The imaging data were evaluated by two radiologists performed single-blind evaluation of tumor volume. When the two had different opinions, a third radiologist reviewed them. The TNM staging criteria for patients were based on the Union for International Cancer Control/American Joint Committee on Cancer (UICC/AJCC) 8th Edition. ${ }^{15}$ The inclusion criteria were 1) 18-75 years old and KPS score $\geq 70$; 2) NSCLC diagnosed by histopathology; 3) no EGFR/ALK/ROS1 mutations; 4) if EGFR/ALK/ROS1 mutation, patients must have received EGFR/ALK/ ROS1-TKIs and exhibited resistance or intolerance; 5) received at least two chemotherapy regimens or intolerance, 6) BM diagnosed by computed tomography/magnetic resonance imaging (CT/MRI); 7) patients received CRT and anlotinib concurrent treatment, or CRT treatment alone; 8) anlotinib was maintained until the patient became intolerant or the disease progressed (Figure 1). ${ }^{16}$ The exclusion criteria were 1) diagnosis with a previous malignancy and the disease was not cured, or presence of other concomitant malignant disease; 2) CRT or concurrent treatment not completed; 3) previously received CRT treatment; 4) received anlotinib treatment before CRT. According to the treatment process, all collected patients 
588 cases with lung cancer treated by radiotherapy
144 cases with lung cancer treated by radiotherapy plus anlotinib

Included:

1. 18-75 years old and KPS score $\geq 70$;

2. Lung cancer with squamous or adenocarcinoma was diagnosed by histopathology;

3. No EGFR/ALK/ROS1 mutations;

4. If EGFR/ALK/ROS1 mutation, patients must receive EGFR/ALK/ROS1-TKIs, and appear resistance or intolerance;

5. Receive at least two chemotherapy regimens or intolerance;

6. Brain metastasis was diagnosis by $\mathrm{CT} / \mathrm{MRI}$;

7. CRT and anlotinib concurrent treatment, or CRT alone.

8. Anlotinib was maintained until the patient is intolerable or the disease progresses.

Excluded:

1. The patient had a previous malignancy and the disease has not been cured, or other concomitant malignant tumor;

2. CRT or concurrent treatment not completed;

3. Who once had CRT;

4. Patients received anlotinib before CRT.

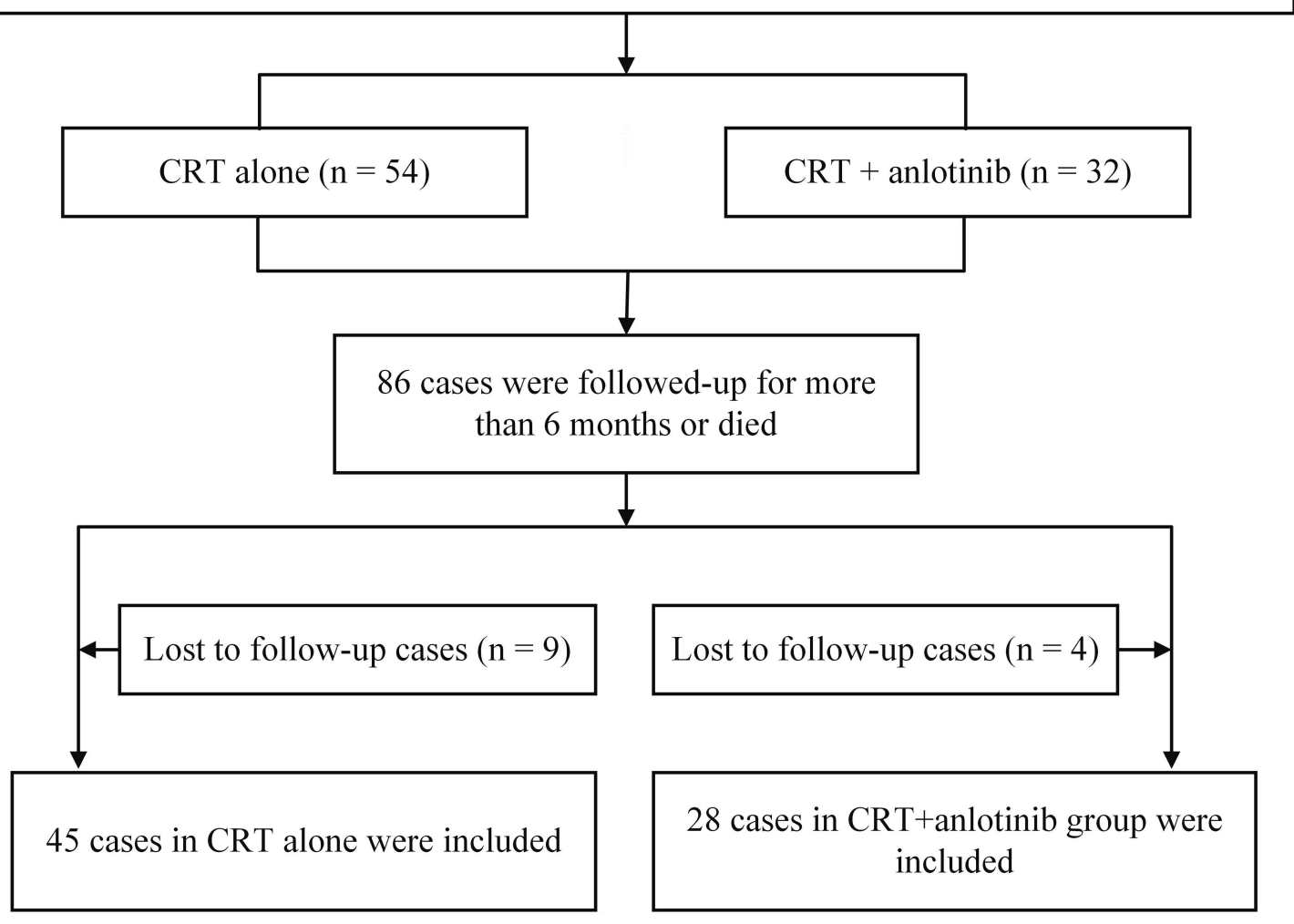

Figure I The flow diagram of included patients. 
were divided into two groups: CRT combined with anlotinib concurrent therapy group (CRT + anlotinib group) or CRT alone group.

\section{Treatment}

Anlotinib treatment was performed according to the drug guidelines of 8-12 mg daily (recommended dose) for 14 days orally and then 7 days off. ${ }^{10}$ The CRT treatment (6 MV X-ray) was the first CRT treatment for all patients. Fifteen patients received intensity modulated radiotherapy (IMRT), 43 patients received conformal radiotherapy, and 15 patients received IMRT for local lesions and conformal radiotherapy for whole brain. CRT treatment included whole-brain radiotherapy (WBRT), WBRT plus local CRT (LCRT), or LCRT, as decided by the multidisciplinary team based on the number of BM, patient KPS score, pathological type, and other factors. BM with $\leq 3$ lesions were mainly assigned to LCRT, and $>3$ lesions were mainly assigned to WBRT or WBRT + LCRT treatments. For CRT, the dose for WBRT was 30-40 Gy in 10-20 fractions. The dose for LCRT was 25-54 Gy in 5-27 fractions. The dose for WBRT + LCRT was 30-40 Gy for WBRT and 10-24 Gy for LCRT. Clinical follow-up was carried out every 3-6 months, and included imaging, physical, and routine laboratory tests. The therapeutic effect was evaluated according to the Response Evaluation Criteria in Solid Tumors (RECIST) 1.1. ${ }^{17}$

\section{Outcomes}

Overall response rate (ORR) was defined as the proportion of complete response (CR) and partial response (PR) cases relative to the total number of evaluable cases. OS was defined based on the initiation of CRT to the death time or last follow-up time. ${ }^{18,19}$ The intracranial PFS (iPFS) and extracranial PFS (ePFS) were defined from the initiation of CRT to intracranial/extracranial progression time or death time, or the last follow-up time for patients who showed no progress or died. Systematic PFS (sPFS) was defined from the initiation of CRT to death, or tumor progression, or the last follow-up time for patients who showed no progress or died. ${ }^{12}$ The last follow-up time was December 2020. The primary endpoints included iPFS and OS, and the secondary endpoints included ePFS and sPFS.

\section{Statistical Analysis}

Patient characteristics were expressed as categorical variables and analyzed by Pearson's chi-square test or Fisher's exact test. The age as a patient characteristic was calculated as mean \pm standard deviation (S.D). Differences in PFS and OS between CRT + anlotinib group and CRT alone group were compared using Cox proportional hazards models. Subgroup analyses in PFS and OS were accomplished by randomized stratification factors and stratified Cox proportional hazards models. Statistical analyses were carried out using SPSS 25.0 (International Business Machines Corporation, Armonk, New York, USA). The figures were prepared using GraphPad Prism v8.3 (GraphPad Software Inc., San Diego, USA). A value of $P<0.05$ with 2 sides was defined as statistical significance.

\section{Results \\ Patient Characteristics}

According to the included and excluded criteria, 86 NSCLC patients with CRT and non-EGFR/ALK/ROS1TKIs indication were included in this retrospective study. Thirteen cases lacking sufficient follow-up data were excluded (Figure 1). Finally, 73 patients were included in the study, including 14 cases of squamous carcinoma $(19.18 \%)$, and 59 cases of adenocarcinoma (80.82\%). The median and average ages of all patients were 57 and 58.5 years (range $30-75$ years), respectively. Of these, 39 patients $(53.42 \%)$ were male and 52 patients $(71.23 \%)$ were never smokers. The KPS scores of 48 patients $(65.75 \%)$ were in the range of $90-100$, and scores of 25 patients $(34.25 \%)$ were in the range of $70-80$. The left lung was the primary cancer site for 38 patients $(52.05 \%)$, and the right lung was the primary cancer site for the other 35 patients (47.95\%). There were 5 (6.85\%), 16 (21.92\%), $11(15.07 \%)$, and $21(28.77 \%)$ of patients classified as stage $\mathrm{T} 1, \mathrm{~T} 2, \mathrm{~T} 3$, and $\mathrm{T} 4$, respectively; and the classifications for the remaining 20 patients $(27.40 \%)$ were not available. There were 4 (5.48\%), 7 (9.59\%), 31 (42.47\%) and $17(23.29 \%)$ patients classified as stage N0, N1, N2, and $\mathrm{N} 3$; the remaining 14 patients (19.18\%) lacked classification data. A total of 39 patients $(53.43 \%)$ had extracranial distant metastasis, and the presence of extracranial distant metastasis was not assessed for 7 patients $(9.60 \%)$. Fourteen patients had the EGFR gene mutation; these patients exhibited resistance to treatment with EGFR/ ALK/ROS1-TKIs and started CRT after BM diagnosis. There were $16,12,33,7$, and 4 patients that received zero-line, first-line, second-line, third-line and fourth-line treatments before CRT, with no significant differences in 
the baseline characteristics $(P>0.05)$. The patient baseline characteristics of the CRT alone and the anlotinib + CRT groups are listed in Table 1 . Of the 73 patients, 28 cases received anlotinib plus CRT, and the other 45 cases received CRT alone.

\section{Efficacy}

The median and mean follow-up time of all patients were 8.0 and 9.82 months, respectively. The ORR values of the CRT + anlotinib group and the CRT alone group were $89.29 \%$ and $80.0 \%$, respectively. Of the 45 patients in the CRT alone group, six patients $(13.33 \%)$ were alive with no evidence of disease progression, 32 patients (71.11\%) died with intracranial progression, 24 patients $(53.33 \%)$ died with extracranial progression, and four patients $(8.89 \%)$ were alive with detected intracranial progression. Of the 28 patients in the CRT + anlotinib group, four patients $(14.29 \%)$ were alive without evidence of disease progression, 15 patients $(53.57 \%)$ were dead with intracranial progression, 13 patients $(46.43 \%)$ were dead with extracranial progression, and three patients $(10.71 \%)$ were alive with intracranial progression. For the whole group, the median iPFS (miPFS) and mOS were 6.0 and 8.0 months, respectively. The miPFS values were 11.0 months for the anlotinib + CRT group and 3.0 months for the CRT alone group (HR $0.52,95 \%$ CI $0.27-0.99, P$ $=0.048$ ) (Figure 2). This indicated that plus anlotinib treatment was closely associated with a significantly longer iPFS when combined with CRT. The mOS of the anlotinib + CRT group was longer than that of the CRT alone group ( 8.5 vs 6.0 months), although this difference was not statistically significant (HR $0.92,95 \%$ CI 0.52 $1.62, P=0.77)$. The CRT + anlotinib group vs CRT alone group was 12.0 vs 13.0 months for median ePFS (mePFS, HR $0.95,95 \%$ CI $0.47-1.94, P=0.89$ ) and 8.0 vs 4.5 months for median sPFS (msPFS, HR $0.85,95 \%$ CI 0.47 $1.85, P=0.85)$. There was no significant difference for ePFS and sPFS in these two groups.

A value of $P<0.1$ was considered a significant difference for univariate analysis. The analysis revealed that iPFS was significantly related to KPS score, pathological type, age, and plus anlotinib treatment (Table 2). OS was related to age, gender, KPS score, and pathological type.

The characteristics identified as significant by univariate analysis were then subjected to adjusted Cox multivariate analyses to analyze the correlation between these characteristics and iPFS or OS. In multivariate analyses, only plus anlotinib significantly prolonged iPFS (HR 0.51,
95\% CI $0.27-0.95, P=0.04$ ) (Figure $3 \mathrm{~A}$ ). Age $\geq 57$ years (HR 1.04, 95\% CI 1.01-1.08, $P=0.014$ ) can significantly decreased OS and KPS score $\geq 90$ (HR $0.59,95 \%$ CI 0.34 $1.05, P=0.04)$ correlated with significantly prolonged OS (Figure 3B). No statistically significant differences were observed between gender, smoking history, $\mathrm{T}$ stage, $\mathrm{N}$ stage, primary site, extracranial distant metastasis, number of brain metastases, number of lines of therapy, and iPFS or OS in this study $(P>0.05)$.

Subgroup analyses indicated that iPFS of LCRT + anlotinib group (miPFS 27.0 months) exhibited the strongest benefits of the groups (Figure 4A). Although there was no statistical significance of the effect of LCRT + anlotinib on OS, the mOS of LCRT + anlotinib group was 36 months, higher than the other groups. The second highest iPFS and OS values were for the LCRT group (miPFS 11.0 months; mOS 18.0 months) (Figure 4B). These results suggest that treatment that combined anlotinib with LCRT was better than LCRT alone, although the difference was not statistically significant. The iPFS of the LCRT group (miPFS 11.0 months) was longer than WBRT + LCRT + anlotinib group (miPFS 8.5 months) and WBRT group (miPFS 2.5 months), OS of LCRT group (mOS 18.0 months) was longer than the WBRT + LCRT + anlotinib group (mOS 8.0 months) and WBRT group (mOS 5.5 months). Overall, these results indicated greater importance of CRT pattern for prognosis than supplemental treatment with anlotinib (Figure 4).

\section{Discussion}

The mechanism of BM from lung cancer is complex, but is closely related to angiogenesis. Angiogenesis in metastasizing lesions can develop through multiple signal pathways, and an important one is the VEGF pathway. Studies have found that the expression level of VEGF in tumor is negatively related to poor prognosis. ${ }^{8}$ As a new type of small molecule and multi-targeting TKI, anlotinib mainly acts through the anti-VEGF pathway for anti-tumor effect. ${ }^{8}$ In 2020 and 2021, oncologists suggested that anlotinib has intracranial activity and can control intracranial tumors. ${ }^{12,20,21}$ The ALTER0303 study also showed that anlotinib can prolong PFS in lung cancer patients with $\mathrm{BM}^{12}$ Anlotinib normalizes the blood vessels in a metastatic tumor, adjusts the internal microenvironment of the tumor, restores the normal permeability of blood vessels, and acts synergistically with CRT to enhance radiosensitivity and reduce brain edema. However, there is no evidence shown that CRT can enhance anlotinib cross the 
Table I Clinical Baseline Characteristics of Included Patients

\begin{tabular}{|c|c|c|c|}
\hline Characteristic & CRT Alone $(n=45)$ & CRT +Anlotinib $(n=28)$ & $P$ value \\
\hline \multicolumn{4}{|l|}{ Age (years) } \\
\hline Average (mean $\pm \mathrm{SD})$ & $57.4 \pm 8.87$ & $60.29 \pm 10.04$ & 0.33 \\
\hline Median & 56 & 63.5 & \\
\hline Range & $4 I-73$ & $30-75$ & \\
\hline \multicolumn{4}{|l|}{ Gender } \\
\hline Female & 21 (46.67\%) & $13(46.43 \%)$ & 0.98 \\
\hline Male & $24(53.33 \%)$ & $15(53.57 \%)$ & \\
\hline \multicolumn{4}{|l|}{ KPS score } \\
\hline $90-100$ & $28(62.22 \%)$ & 20 (7I.43\%) & 0.42 \\
\hline $70-80$ & $17(37.78 \%)$ & $8(28.57 \%)$ & \\
\hline \multicolumn{4}{|l|}{ Smoking history } \\
\hline Yes & II (24.44\%) & 10 (35.7I\%) & 0.3 \\
\hline No & $34(75.56)$ & $18(64.29 \%)$ & \\
\hline \multicolumn{4}{|l|}{ Primary site } \\
\hline Left & $22(48.89 \%)$ & $16(57.14 \%)$ & 0.49 \\
\hline Right & $23(51.11 \%)$ & $12(42.86 \%)$ & \\
\hline \multicolumn{4}{|l|}{ Pathological type } \\
\hline Adenocarcinoma & 38 (84.44\%) & $21(75 \%)$ & 0.32 \\
\hline Squamous carcinoma & 7 (15.56\%) & 7 (25\%) & \\
\hline \multicolumn{4}{|l|}{ T stage } \\
\hline TI & I (2.22\%) & 4 (14.29\%) & 0.11 \\
\hline $\mathrm{T} 2$ & $9(20 \%)$ & 7 (25\%) & \\
\hline T3 & 7 (15.56\%) & 4 (14.29\%) & \\
\hline $\mathrm{T} 4$ & $17(37.78 \%)$ & $4(14.29 \%)$ & \\
\hline Tx & II (24.44\%) & $9(32.14 \%)$ & \\
\hline \multicolumn{4}{|l|}{$\mathrm{N}$ stage } \\
\hline No & $2(4.44 \%)$ & $2(7.14 \%)$ & 0.41 \\
\hline $\mathrm{NI}$ & $4(8.89 \%)$ & $3(10.71 \%)$ & \\
\hline N2 & $23(51.11 \%)$ & $8(28.57 \%)$ & \\
\hline N3 & 8 (17.78\%) & $9(32.14 \%)$ & \\
\hline $\mathrm{Nx}$ & 8 (I7.91\%) & $6(21.43 \%)$ & \\
\hline \multicolumn{4}{|l|}{ Brain metastases } \\
\hline$\leq 3$ & $10(22.22 \%)$ & $8(28.57 \%)$ & 0.54 \\
\hline$>3$ & 35 (77.78\%) & 20 (7I.43\%) & \\
\hline \multicolumn{4}{|l|}{ CRT pattern } \\
\hline WBRT & $30(66.67 \%)$ & $13(46.43 \%)$ & 0.16 \\
\hline WBRT + LCRT & $8(17.78 \%)$ & 7 (25.0\%) & \\
\hline LCRT & 7 (15.55\%) & 8 (28.57\%) & \\
\hline \multicolumn{4}{|c|}{ Extracranial distant metastasis } \\
\hline Yes & $24(53.33 \%)$ & I5 (53.57\%) & 0.84 \\
\hline No & $16(35.56 \%)$ & II (39.29\%) & \\
\hline Not available & $5(11.11 \%)$ & $2(7.14 \%)$ & \\
\hline
\end{tabular}

(Continued) 
Table I (Continued).

\begin{tabular}{|l|c|c|c|}
\hline Characteristic & CRT Alone (n = 45) & CRT +Anlotinib (n = 28) & P value \\
\hline Treatment-line & & & \\
Zero-Line & $8(17.78 \%)$ & $8(28.57 \%)$ & 0.084 \\
First-Line & $4(8.89 \%)$ & $8(28.57 \%)$ \\
Second-Line & $25(55.56 \%)$ & $8(28.57 \%)$ \\
Third-Line & $5(11.11 \%)$ & $2(7.14 \%)$ \\
Fourth-Line & $2(4.44 \%)$ & $2(7.14 \%)$ \\
\hline
\end{tabular}

Abbreviations: CRT, cranial radiotherapy; KPS, Karnofsky Performance Status; WBRT, whole brain radiotherapy; LCRT, local cranial radiotherapy.

$\mathrm{BBB}$, and the detailed mechanism of anlotinib action in lung cancer patients with BM requires further study. ${ }^{13}$ Additionally, further clinical studies are required to determine whether the combination of anlotinib and CRT is better than CRT alone for patients with BM from lung cancer who failed to respond to multi-line chemotherapy or without EGFR/ALK/ROS1-TKIs indication.

In our study, CRT + anlotinib treatment was significantly superior to treatment with CRT alone (miPFS: 11.0 vs 3.0 months, $P=0.048)$. However, this treatment did not obviously improve OS and $\mathrm{sPFS}(P>0.05)$, although
mOS and msPFS of CRT + anlotinib group were longer than those of the CRT alone group. These results were consistent with those reported for the ALTER0303 study, where anlotinib affected PFS but did not significantly prolong OS in patients with BM. In our study, intracranial control was more effective than systemic control, which is likely related to CRT mainly acting to control intracranial lesions. Univariate analysis and multivariate analysis of clinic baseline characteristics and patient survival data showed that plus anlotinib was an independent prognostic factor to improve iPFS $(P<0.05)$, and younger age and
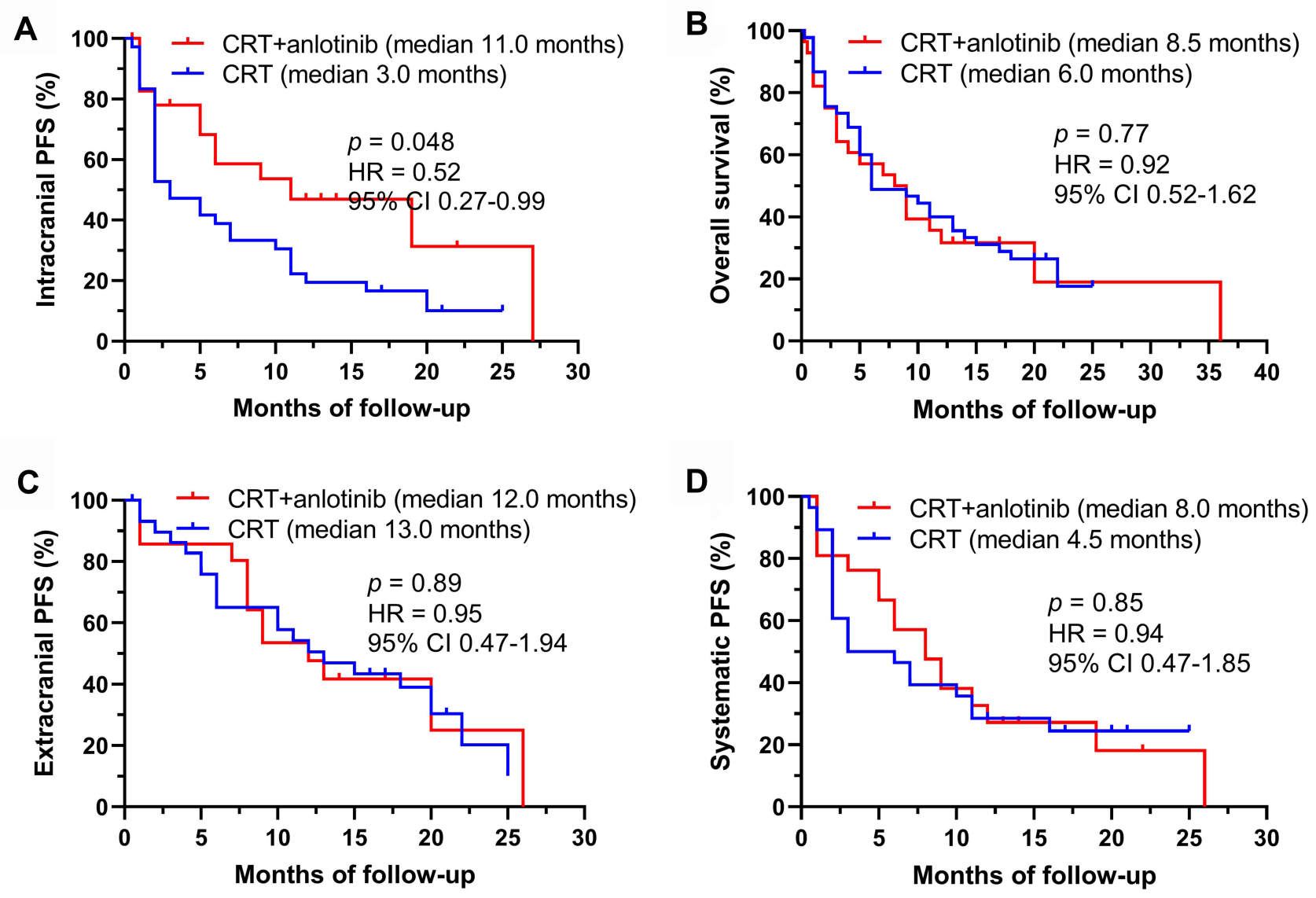

Figure 2 The survival analysis of different treatment groups. (A) iPFS, (B) OS, (C) ePFS and (D) sPFS for patients with BM at baseline. 
Table 2 Univariate Analysis Between Different Characteristics and iPFS or OS

\begin{tabular}{|c|c|c|c|c|}
\hline \multirow[t]{2}{*}{ Variable } & \multicolumn{2}{|c|}{ iPFS } & \multicolumn{2}{|c|}{ os } \\
\hline & HR (95\% Cl) & $P$ value & HR (95\% Cl) & $P$ value \\
\hline \multicolumn{5}{|l|}{ Group } \\
\hline CRT + Anlotinib vs CRT & $0.58(0.32-1.03)$ & 0.06 & I.08 (0.63-I.87) & 0.77 \\
\hline \multicolumn{5}{|l|}{ Gender } \\
\hline Female vs Male & $1.37(0.8 \mathrm{I}-2.32)$ & 0.24 & $1.65(0.96-2.84)$ & 0.07 \\
\hline \multicolumn{5}{|l|}{ Age } \\
\hline$\geq 57$ years vs $<57$ years & $1.03(1-1.06)$ & 0.05 & $1.04(1.01-1.08)$ & 0.01 \\
\hline \multicolumn{5}{|l|}{ KPS score } \\
\hline $90-100$ vs $70-80$ & $0.53(0.3|-0.9|)$ & 0.02 & $0.52(0.30-0.90)$ & 0.02 \\
\hline \multicolumn{5}{|l|}{ Smoking history } \\
\hline Yes vs No & $1.63(0.92-2.89)$ & 0.1 & I.54 (0.87-2.7I) & 0.14 \\
\hline \multicolumn{5}{|l|}{ Pathological type } \\
\hline Squamous vs adenocarcinoma & $0.56(0.29-1.1)$ & 0.09 & $0.47(0.24-0.90)$ & 0.02 \\
\hline \multicolumn{5}{|l|}{ T stage } \\
\hline Tx & 1 & & - & \\
\hline TI & $0.66(0.22-2)$ & 0.47 & $0.50(0.14-1.7 \mid)$ & 0.27 \\
\hline $\mathrm{T} 2$ & $0.79(0.38-1.66)$ & 0.53 & I.0I $(0.48-2.10)$ & 0.99 \\
\hline T3 & $0.68(0.3-1.53)$ & 0.35 & $0.76(0.33-1.72)$ & 0.51 \\
\hline $\mathrm{T} 4$ & $0.54(0.27-1.1)$ & 0.1 & $0.65(0.31-1.34)$ & 0.24 \\
\hline \multicolumn{5}{|l|}{$N$ stage } \\
\hline $\mathrm{Nx}$ & I & & - & \\
\hline No & $0.27(0.05-1.36)$ & 0.11 & $0.31(0.07-1.37)$ & 0.12 \\
\hline $\mathrm{NI}$ & $0.88(0.26-2.92)$ & 0.83 & $0.69(0.24-2.01)$ & 0.5 \\
\hline N2 & $0.9(0.26-3.16)$ & 0.87 & $0.96(0.32-2.9)$ & 0.94 \\
\hline N3 & $0.86(0.24-3.11)$ & 0.82 & $0.74(0.23-2.36)$ & 0.66 \\
\hline \multicolumn{5}{|l|}{ Primary site } \\
\hline Left vs right & $1.28(0.75-2.17)$ & 0.36 & $0.74(0.43-1.27)$ & 0.27 \\
\hline \multicolumn{5}{|l|}{ Number of brain metastases } \\
\hline$>3$ vs $\leq 3$ & $1.22(0.58-2.57)$ & 0.61 & I.83 (0.78-4.27) & 0.16 \\
\hline \multicolumn{5}{|l|}{ Extracranial distant metastasis } \\
\hline Yes vs No & $1.14(0.64-2.02)$ & 0.66 & I.I (0.62-1.96) & 0.75 \\
\hline \multicolumn{5}{|l|}{ Treatment-line } \\
\hline Zero-Line & I & & I & \\
\hline First-Line & $0.93(0.26-3.33)$ & 0.91 & $0.76(0.24-2.37)$ & 0.63 \\
\hline Second-Line & $1.78(0.49-6.46)$ & 0.38 & $0.91(0.28-2.97)$ & 0.88 \\
\hline Third-Line & $1.32(0.4-4.37)$ & 0.65 & $0.91(0.32-2.64)$ & 0.87 \\
\hline Fourth-Line & $0.48(0.1-2.38)$ & 0.37 & $0.61(0.15-2.43)$ & 0.48 \\
\hline
\end{tabular}

Abbreviations: OS, overall survival; iPFS, intracranial progression free survival; CRT, cranial radiotherapy; KPS, Karnofsky Performance Status; HR, hazard ratio; Cl, confidence interval.

higher KPS score were independent prognostic factors to improve OS $(P<0.05)$. This may be because the good physical condition of a patient can influence the effectiveness of treatment. The subgroup analysis of survival data showed that LCRT + anlotinib treatment of lung cancer patients improved and extended iPFS and OS compared to those of the other treatment groups, but there was no significant difference in the treatment pattern $(P>0.05)$. 


\section{Multivariate analysis for iPFS}

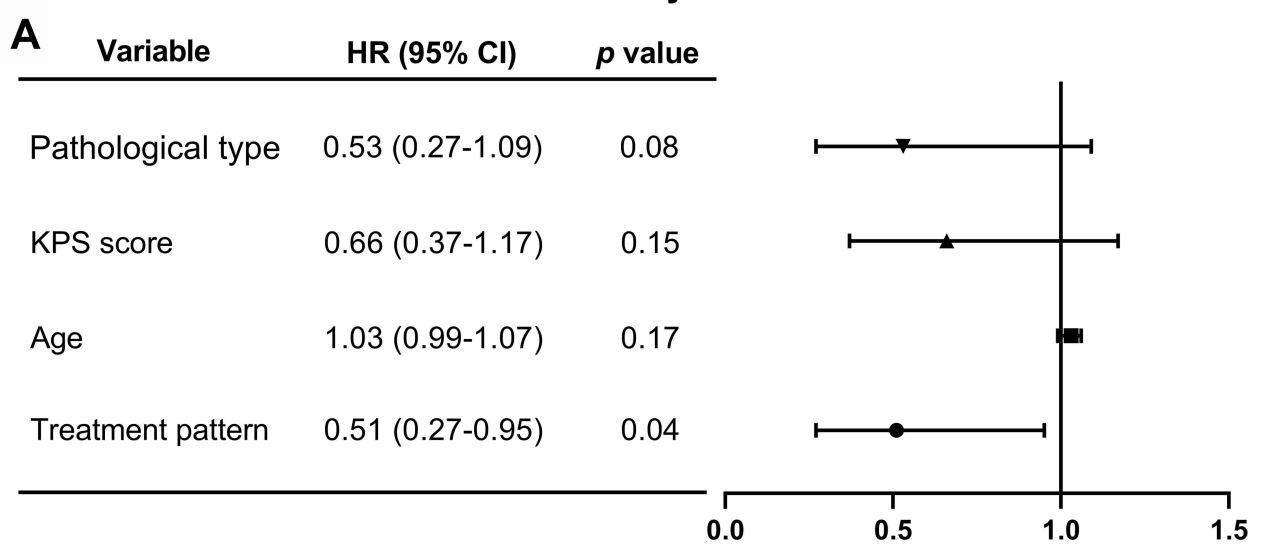

B Multivariate analysis for OS

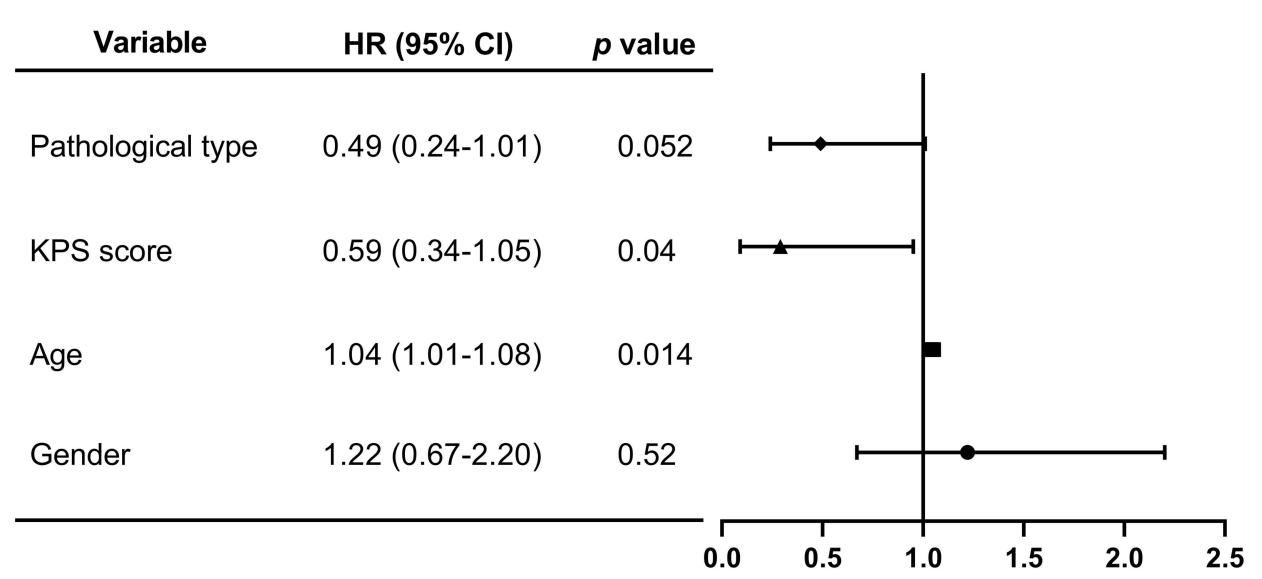

Figure 3 After univariate analysis, the significant variables were chosen for multivariate analysis for iPFS and OS. In multivariate analysis, (A) only the plus anlotinib treatment was positively correlated with prolonged iPFS $(P<0.05)$; (B) age $<57$ years and KPS score $\geq 90$ were positively correlated with prolonged OS $(P<0.05)$.

This suggests that the addition of anlotinib may be the most beneficial for LCRT patients, and this effect should be studied further.

Our study is limited in that it is a retrospective study, which does not allow randomization of patients and affects homogeneity, thus reducing the level of evidence. Another limit is that the number of cases analyzed was less than ideal. Therefore, future studies should utilize an expanded sample size, conduct prospective research, and eliminate heterogeneity to be able to draw more vigorous conclusions. Despite these limitations, our study can serve as strategic reference for the current clinical treatment of
NSCLC patients with BM and non-EGFR/ALK/ROS1TKIs indications.

\section{Conclusion}

In this study, we analyzed the efficacy of anlotinib (multitarget inhibitors) combined with CRT for patients with BM from advanced NSCLC with non-EGFR/ALK/ROS1-TKIs indications. The results indicated that the concurrent use of anlotinib has obvious clinical value to prolong the iPFS of patients with CRT. Our study has important reference significance for the clinical treatment of $\mathrm{BM}$ from NSCLC with non-EGFR/ALK/ROS1-TKIs indications. 
A

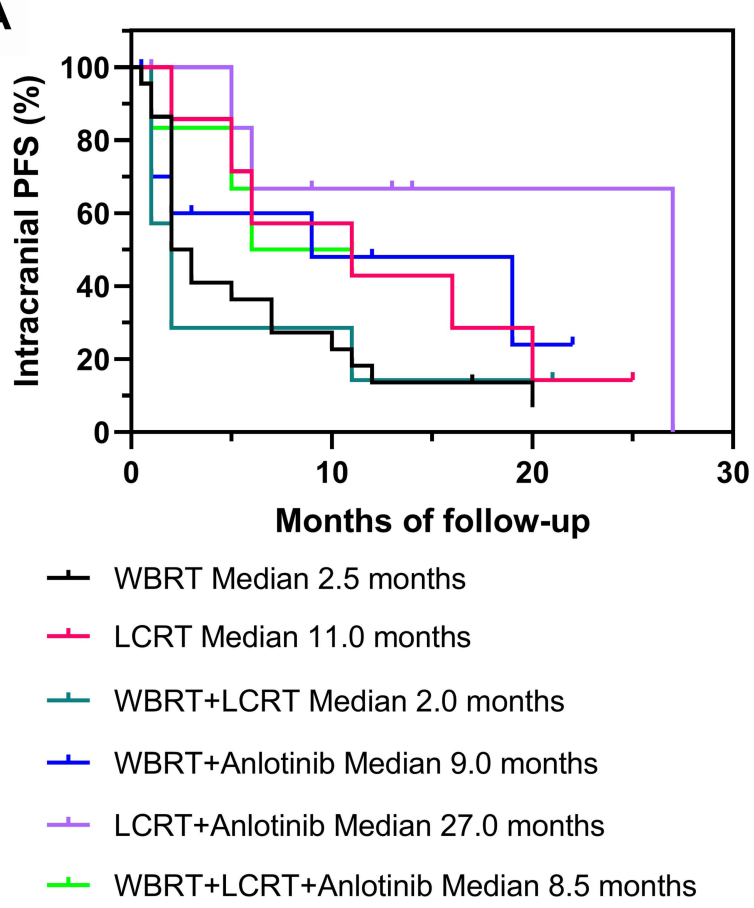

B

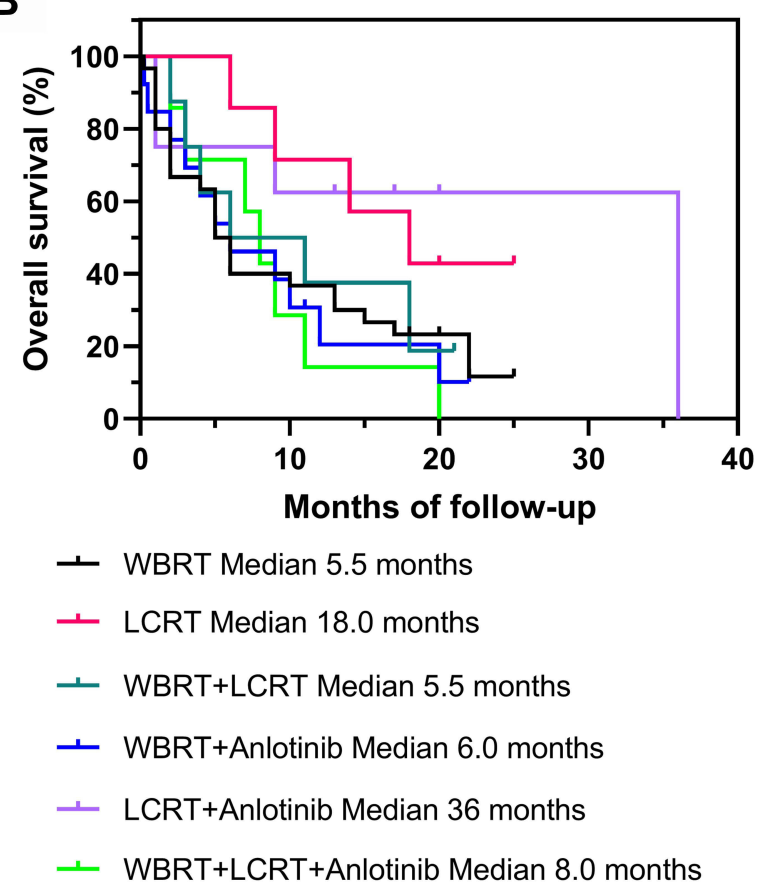

Figure 4 The subgroup analysis of different treatment patterns for BM patients at baseline. (A) iPFS and (B) OS of CRT alone group and CRT + anlotinib group for patients with different treatment patterns.

\section{Abbreviations}

NSCLC, non-small cell lung cancer; BM, brain metastasis; mOS, median survival time; OS, survival time; TKIs, tyrosine kinase inhibitors; ALK, anaplastic lymphoma kinase; EGFR, epidermal growth factor receptor; ROS1, C-ros oncogene 1-receptor tyrosine kinase; CR, complete remission; mPFS, median progression-free survival; TTF, time to treatment failure; PFS, progression-free survival; BBB, blood-brain barrier; HR, hazard ratio; CI, confidence interval; ORR, objective response rate; TTBP, time to brain progression; CRT, cranial radiotherapy; KPS, Karnofsky Performance Status; UICC, Union for International Cancer Control; AJCC, American Joint Committee on Cancer; CT/MRI, computed tomography/ magnetic resonance imaging; WBRT, whole-brain radiotherapy; RECIST, Response Evaluation Criteria in Solid Tumors; ORR, Overall response rate; PR, partial response; iPFS, intracranial progression-free survival; ePFS, extracranial progression-free survival; sPFS, Systematic progression-free survival; S.D, standard deviation; miPFS, median intracranial progression-free survival; mePFS, median extracranial progression-free survival; msPFS, median systematic progression-free survival.

\section{Data Sharing Statement}

The data used and/or analyzed in this study are available from the corresponding author upon reasonable request.

\section{Ethics Approval and Consent to Participate}

This study was a retrospective study, which utilized collected clinical data of patients, did not interfered with the treatment plan of patients, did not present physiological risks to patients, and maintained the privacy of patients. Some data were for patients who had died before this study. The ethics committee of the First Affiliated Hospital of Bengbu Medical College considered the riskbenefits and determined that informed consent was not required for this analysis. All processes conformed to the Declaration of Helsinki. The study design was reviewed and approved by the First Affiliated Hospital of Bengbu Medical College.

\section{Acknowledgments}

The study was supported by the First Affiliated Hospital of Bengbu Medical College Science Fund for Distinguished 
Young Scholars (No. 2019BYYFYJQ04), the Natural Science Foundation of Bengbu Medical College (No. BYKY2019097ZD), the Bengbu Medical College Science Fund for "Excellent Young Teachers in 512 Talent Development Programme" (No. BY51201314), and the Bengbu - Bengbu Medical College Joint Research Project (No. BYLK201810).

\section{Disclosure}

The authors report no conflicts of interest in this work.

\section{References}

1. Siegel RL, Miller KD, Jemal A. Cancer statistics, 2020. CA Cancer J Clin. 2020;70(1):7-30. doi:10.3322/caac.21590

2. Zhang H, Wang A, Tan Y, et al. NCBP1 promotes the development of lung adenocarcinoma through up-regulation of CUL4B. J Cell Mol Med. 2019;23(10):6965-6977. doi:10.1111/jcmm.14581

3. He Z, Shi Z, Sun W, et al. Hemocompatibility of folic-acid-conjugated amphiphilic PEG-PLGA copolymer nanoparticles for co-delivery of cisplatin and paclitaxel: treatment effects for non-small-cell lung cancer. Tumour Biol. 2016;37(6):7809-7821. doi:10.1007/s13277-0154634-1

4. Sung H, Ferlay J, Siegel RL, et al. Global cancer statistics 2020: GLOBOCAN estimates of incidence and mortality worldwide for 36 cancers in 185 countries. CA Cancer J Clin. 2021;71(3):209-249. doi: $10.3322 /$ caac. 21660

5. Yang S, Zhang Z, Wang Q. Emerging therapies for small cell lung cancer. J Hematol Oncol. 2019;12(1):47. doi:10.1186/s13045-0190736-3

6. Yang ZR, Liu MN, Yu JH, et al. Treatment of stage III non-small cell lung cancer in the era of immunotherapy: pathological complete response to neoadjuvant pembrolizumab and chemotherapy. Transl Lung Cancer Res. 2020;9(5):2059-2073. doi:10.21037/tlcr-20-896

7. Alcibar OL, Nadal E, Romero Palomar I, Navarro-Martin A. Systematic review of stereotactic body radiotherapy in stage III nonsmall cell lung cancer. Transl Lung Cancer Res. 2021;10(1):529-538. doi: $10.21037 /$ tlcr-2020-nsclc-04

8. Shen G, Zheng F, Ren D, et al. Anlotinib: a novel multi-targeting tyrosine kinase inhibitor in clinical development. $J$ Hematol Oncol. 2018;11(1):120. doi:10.1186/s13045-018-0664-7

9. Si X, Zhang L, Wang H, et al. Quality of life results from a randomized, double-blinded, placebo-controlled, multi-center Phase III trial of anlotinib in patients with advanced non-small cell lung cancer. Lung Cancer. 2018;122:32-37. doi:10.1016/j.lungcan.2018.05.013
10. Si X, Zhang L, Wang H, et al. Management of anlotinib-related adverse events in patients with advanced non-small cell lung cancer: experiences in ALTER-0303. Thorac Cancer. 2019;10(3):551-556. doi:10.1111/1759-7714.12977

11. Han B, Li K, Wang Q, et al. Effect of anlotinib as a third-line or further treatment on overall survival of patients with advanced nonsmall cell lung cancer: the ALTER 0303 phase 3 randomized clinical trial. JAMA Oncol. 2018;4(11):1569-1575. doi:10.1001/ jamaoncol.2018.3039

12. Jiang S, Liang H, Liu Z, et al. The impact of anlotinib on brain metastases of non-small cell lung cancer: post hoc analysis of a phase III randomized control trial (ALTER0303). Oncologist. 2020;25 (5):870-874. doi:10.1634/theoncologist.2019-0838

13. Brun L, Dupic G, Chassin V, et al. Hypofractionated stereotactic radiotherapy for large brain metastases: optimizing the dosimetric parameters. Cancer Radiother. 2021;25(1):1-7. doi:10.1016/j. canrad.2020.04.011

14. Kahrom A, Grimley R, Jeffree RL. A case of delayed cyst formation post brain AVM stereotactic radiosurgery for arteriovenous malformation: case report. J Clin Neurosci. 2021;87:17-19. doi:10.1016/j. jocn.2021.01.051

15. Lancia A, Merizzoli E, Filippi AR. The 8(th) UICC/AJCC TNM edition for non-small cell lung cancer staging: getting off to a flying start? Ann Transl Med. 2019;7(Supp16):S205. doi:10.21037/ atm.2019.07.02

16. Duan Y, Qian J, Chen K, Zhang Z. Necessity of adjuvant postoperative radiotherapy for diffuse pigmented villonodular synovitis of the knee: a case report and literature review. Medicine. 2018;97(3): e9637. doi:10.1097/md.0000000000009637

17. He Z, Zhang H, Cui Z, et al. A case of chemorefractory metastatic type AB thymoma sensitive to helical tomotherapy. Ann Transl Med. 2019;7(12):274. doi:10.21037/atm.2019.05.34

18. Han B, Li K, Zhao Y, et al. Anlotinib as a third-line therapy in patients with refractory advanced non-small-cell lung cancer: a multicentre, randomised phase II trial (ALTER0302). Br J Cancer. 2018;118(5):654-661. doi:10.1038/bjc.2017.478

19. Li Y, Liu J, Li HX, et al. Radiomics signature facilitates organ-saving strategy in patients with esophageal squamous cell cancer receiving neoadjuvant chemoradiotherapy. Front Oncol. 2020;10:615167. doi: $10.3389 /$ fonc. 2020.615167

20. Gao XH, Zheng J, Ma L, et al. Mitigation of acute radiation-induced brain injury in a mouse model using anlotinib. Ann Palliat Med. 2021;10(1):312-322. doi:10.21037/apm-20-2284

21. Wang Y, Liang D, Chen J, et al. Targeted therapy with anlotinib for a patient with an oncogenic FGFR3-TACC3 fusion and recurrent glioblastoma. Oncologist. 2021;26(3):173-177. doi:10.1002/onco.13530
Cancer Management and Research

\section{Publish your work in this journal}

Cancer Management and Research is an international, peer-reviewed open access journal focusing on cancer research and the optimal use of preventative and integrated treatment interventions to achieve improved outcomes, enhanced survival and quality of life for the cancer patient.
The manuscript management system is completely online and includes a very quick and fair peer-review system, which is all easy to use. Visit http://www.dovepress.com/testimonials.php to read real quotes from published authors. 